\title{
CONDICIONES TÉMPORO-ESPACIALES DE ADOLESCENTES QUE HABITAN EN UNA COPROPIEDAD HORIZONTAL DE LA CIUDAD DE BARRANQUILLA, COLOMBIA
}

\section{TEMPORO-SPATIAL CONDITIONS OF ADOLESCENT LIVING IN HORIZONTAL CO-OWNERSHIP BUILDING AT BARRANQUILLA, COLOMBIA}

\author{
Armando Aroca Araújo ${ }^{1}$, Lina Álvarez Toro ${ }^{2}$, María Alejandra Mejía Pérez ${ }^{3}$
}

${ }^{1}$ Doctorando en Educación énfasis en Educación Matemática. Universidad Francisco José de Caldas. Profesor Asociado. Universidad del Atlántico, Calle 74 No. 38-100, Apto 901, Bloque 3, Conjunto Residencial Altos de Betania, Barranquilla, Colombia, e-mail: armandoaroca@mail.uniatlantico.edu.co; ${ }^{2}$ Magister en Desarrollo Infantil. Universidad de Manizales. Profesora Tiempo Completo Ocasional. Universidad del Atlántico, Calle 74 No. 38-100, Apto 901, Bloque 3, Conjunto Residencial Altos de Betania, Barranquilla, Colombia, e-mail: linaalvarez@mail.uniatlantico.edu.co; ${ }^{3}$ Estudiante Licenciatura en Matemáticas. Universidad del Atlántico, Carrera 22C No. 64B-25, Barrio San Felipe, Barranquilla, Colombia, e-mail: mari.ale0425@ live.com

\section{Rev. U.D.C.A Act. \& Div. Cient. 21(1): 217-225, Enero-Junio,2018 https://doi.org/10.31910/rudca.v21.n1.2018.680 \\ RESUMEN \\ conditions of solitude, confinement to the room, addiction to video games and tensions with parents that adolescents have to expand their temporality and spatiality. The methodology used was that of Social Education Nuclei, NES. The strategy used to identify temporomandibular conditions was through the formation of a focal group where 4 adolescents participated. The main findings refer to how the relationships between parents and their adolescent children establish limits to the development of the spatiality and temporality.}

La investigación tuvo como objetivo identificar cuáles son las condiciones témporo-espaciales que tienen los niños, en un rango de edad de 14 a 16 años, residentes en una copropiedad horizontal, que consta de tres Bloques o Edificios residenciales, una administración, áreas comunes, delimitación por paredes y un sistema de vigilancia. El problema que se identificó consiste en las condiciones de soledad, el confinamiento a la habitación, la adicción a los videojuegos y las tensiones con padres que tienen los adolescentes para ampliar su temporalidad y su espacialidad. La metodología empleada fue la de Núcleos de Educación Social -NES-. La estrategia que se empleó para poder identificar las condiciones témporo-espaciales fue a través de la conformación de un grupo focal, donde participaron 4 adolescentes. Los principales hallazgos hacen referencia de cómo las relaciones entre padres de familia y sus hijos adolescentes establecen límites al desarrollo de la espacialidad y la temporalidad.

Palabras clave: Núcleos de Educación Social, Copropiedad Horizontal, temporalidad, espacialidad.

\section{SUMMARY}

This research aimed to identify what are the temporo-spatial conditions that children have in a range of age from 14 years to 16 years when they are residents of a horizontal co-ownership that has three blocks or residential buildings, walls, and a surveillance system. The problem identified is the
Key words: Kernels of Social Education, Horizontal Coparcenary, temporality, spatiality.

\section{INTRODUCCIÓN}

Se pudo demostrar, en Aroca \& Álvarez (2016), que son pocas las investigaciones sobre las condiciones de vida de los niños o de los adolescentes, que residen en Copropiedad Horizontal; no obstante, existen estudios que muestran otras dimensiones de vida de esta población infantil, tales como la de Rincón \& Maldonado (2007), quienes analizaron los motivos que conducen a las familias a vivir en copropiedad horizontal; García \& Peralta (2015) muestran la relación de los condominios cerrados o urbanizaciones multifamiliares cerradas con respecto al espacio exterior y otras investigaciones, como la de Roitman (2003), quien analiza otros aspectos, como segregación social urbana y los barrios cerrados. En Latinoamérica, se han hecho investigaciones sobre el desarrollo urbano de la vivienda, de lo cual, se puede interpretar las condiciones espaciales y temporales que se 
impone a las familias; entre esas investigaciones, están Meyer \& Bärh (2004), Alfonso (2005) y Janoschka \& Borsdorf (2006). Otros autores, como Lasarte \& Feliu (2005), profundizan en las normas de convivencia, la organización social o administrativa de la Copropiedad Horizontal; sin embargo, el tema de las condiciones de desarrollo de espacialidad y de temporalidad de los niños y adolescentes, aún es un campo amplio de investigación.

Entre la bibliografía sobre espacio, espacialidad, tiempo y temporalidad, se identificaron algunos referentes teóricos, afines con esta investigación. Por espacialidad, se comparte que es la toma de conciencia del sujeto sobre el espacio que lo rodea. Por temporalidad, se comparte que es la toma de consciencia del sujeto sobre el tiempo que regula su cotidianidad. Según Garrido (2005), el espacio geográfico es visto como una noción experiencial que es inherente a la vivencia de los sujetos y Blanco (2007) considera que la idea de espacio está asociada con una cierta concepción de los procesos sociales. El espacio socio-geográfico es una herramienta para entender y explicar parte de la realidad, como lo plantea Blanco (2007).

En cuanto a la relación entre tiempo y temporalidad, se retoma como base teórica a Vasco (2000). Para este autor, no se puede hablar ampliamente acerca del tiempo sin especializarlo; se trata de una metáfora obligada por nuestro cuerpo, por nuestras acciones y movimientos. Una primera acepción de la palabra "tiempo" es, pues, la temporalidad como propiedad inherente a todo proceso, relacionado con la dinamicidad. Es aquello que nos permite decir que los procesos fluyen, duran, corren, discurren.

El problema que se abordó en esta investigación fueron las implicaciones que tienen las condiciones témporo-espaciales para el desarrollo de los adolescentes: confinamiento al espacio local, adicciones a video juegos, poco desarrollo de la experiencia socio-geográfica, uso del espacio próximo y externo y condicionamientos de los padres. La clasificación de los espacios responde al espacio propio, la habitación, el espacio local, el apartamento, el espacio próximo, la Unidad Residencial y el espacio externo, por fuera la de la Unidad Residencial.

\section{MATERIALES Y MÉTODOS}

Para el análisis de la información obtenida en esta fase de investigación, se emplearon 31 categorías, a priori que, en la primera fase, fueron emergentes en la investigación desarrollada por Aroca \& Álvarez (2016). Esta primera fase de investigación consistió en el análisis de las condiciones témporo-espaciales de niños, de un rango de edad entre los 8 y 11 años. Estas categorías, se presentan en la columna 1 del cuadro 1. La segunda fase, identificó las categorías para un grupo de 4 adolescentes, en el rango de 14 a 16 años (columna 2) (Cuadro 1).

La investigación, se realizó con 4 adolescentes, en un rango de edad de 14 a 16 años, quienes habitan en una Copropiedad Horizontal o Unidad Residencial de la ciudad de Barranquilla, cuyas características principales son: tiene tres edificios, uno de 14 pisos y los otros dos, de 15 pisos, cada uno; cada piso tiene cuatro apartamentos; en promedio, cada apartamento tiene $100 \mathrm{~m}^{2}$ y están compuestos de tres habitaciones, dos baños, una sala y una cocina; los apartamentos que tienen dos habitaciones son de menor dimensión. La Unidad Residencial cuenta con un reducido parque, una piscina, un pequeño cine, un limitado gimnasio y una sala de juegos, con una mesa de billar, una cancha de micro fútbol y, a la vez, de basquetbol, parqueaderos para visitantes y propietarios de los primeros pisos y un parqueadero subterráneo, solo para propietarios. Con los adolescentes, se organizó un grupo focal (Mella, 2000) y la entrevista semiestructura tuvo una duración de alrededor de una hora y media. Para la transcripción de los audios, se emplearon los signos de transcripción Val.Es.Co. A los adolescentes, se les codificó sus nombres, para guardar su identidad, empleando los códigos A1, A2, A3 y A4.

El principal método aplicado para esta reflexión fue la de Núcleos de Educación Social -NES- (García \& Guerrero, 2012), que se articula en un proceso investigativo por Etapas y Escenarios de Investigación. En esta investigación, se asumieron las etapas Sujeto y Copropiedad Horizontal. El análisis de la información, se basó en la estrategia de fichaje, propuesta para los NES. Esta estrategia permitió establecer cuáles, de las 31 categorías que emergieron con los niños de rango de edad entre los 8 y 11 años, permanecían y si emergieron otras categorías propias, del rango de edad de los 14 a los 16 años.

\section{RESULTADOS Y DISCUSIÓN}

A continuación, se hace la descripción de cada las categorías encontradas en la segunda fase de investigación y que se presentaron en la columna 2 del cuadro 1 . En esta descripción, se hará la comparación con los resultados encontrados en Aroca \& Álvarez (2016).

Número de amigos y posibilidades de jugar: Mientras que para el rango de 8 a 11 años jugar varios juegos era cotidiano, para el rango de 14 a 16 años, conversar, practicar un deporte, estar solo y salir a centros comerciales, era lo prioritario. Tal como lo señala Piedrahíta et al. (2012), los adolescentes recorren los diferentes lugares del centro comercial, prefieren ubicarse en ciertos espacios donde se les observa con mayor frecuencia y que, en cierta forma, convierten en sus territorios. Allí, se encuentran, se reúnen y se 
Cuadro 1. Comparación entre las categorías témporo-espaciales encontradas en rangos de edad de 8 a 11 años y 14 a 16 años.

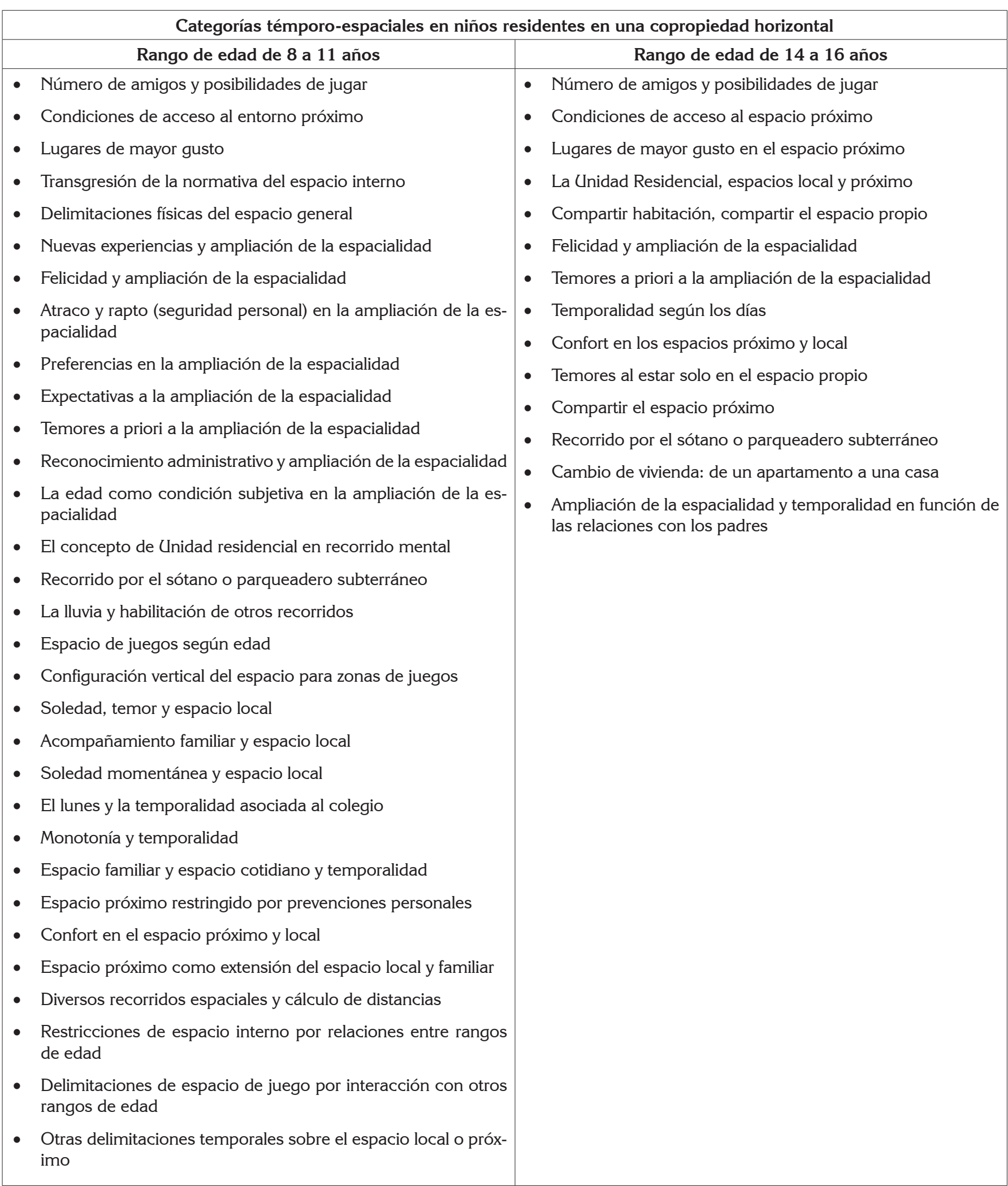


desenvuelven con mayor tranquilidad; estos espacios suelen ser las zonas de juego y los cines. Así, para algunos autores, como Kim et al. (2003), el centro comercial se transforma en instancia predilecta de socialización y satisfacción de necesidades emocionales de adolescentes.

Condiciones de acceso al entorno próximo: En el rango de 14 a 16 años hay libertad plena para acceder al espacio próximo, a diferencia del rango de 8 a 11 años; por ejemplo, se notó sorpresa en algunos de los entrevistados, cuando se indagó por los permisos que ellos debían solicitar para salir del apartamento al resto de la unidad residencial, es decir, los adolescentes participantes no solicitan permisos a sus padres para salir al espacio próximo.

Lugares de mayor gusto en el espacio próximo: El parque y la piscina dejaron de ser los lugares de mayor gusto para el rango de edad en cuestión; no se encontró evidencia sobre algún lugar que genera gusto para frecuentar en el espacio próximo. El parque, se convirtió, para este rango de edad, en un lugar de encuentro para conversar en algunas ocasiones, pero, en especial, para planear actividades, como salidas a centros comerciales y, luego, ejecutarlas. También, se nombró la cancha de micro fútbol y la sala de juegos, donde está la mesa de billar, donde el rango de 8 a 11 años no tiene acceso, caminar algunos sectores e ir a algunos apartamentos. Se interpreta que ellos consideran este espacio, donde manifiestan sentirse a gusto, como un lugar donde pueden hablar tranquilamente y expresarse con libertad, pero es más como un lugar de encuentro para la planeación de salidas y, especialmente, a centros comerciales.

Se pudo evidenciar que los lugares frecuentados por los adolescentes en el espacio próximo se redujeron, con respecto a los niños de rango de 8 a 11 años, lo que demuestra que los adolescentes participantes tienen menos movilidad en el espacio próximo y la reducen a su espacio propio o local.

La Unidad Residencial, espacios local y próximo: Al preguntarles a los adolescentes cuál es el día más aburrido que pasan en la Unidad Residencial, respondieron:

\begin{tabular}{|c|c|c|}
\hline $\begin{array}{l}\text { A1: No/ no } \\
\text { hay ningún día } \\
\text { porque// aquí } \\
\text { está todo aquí } \\
\text { mismo// O sea } \\
\text { aquí esta mis } \\
\text { amigos/ aquí está } \\
\text { el gimnasio / y } \\
\text { para jugar }\end{array}$ & $\begin{array}{l}\text { A2: iNin- } \\
\text { guno! } \\
\text { Armando: } \uparrow \\
\text { ¿Ninguno? } \\
\text { A2: iAcá es } \\
\text { bacano! }\end{array}$ & $\begin{array}{l}\text { A3: Los domingo/ } \\
\text { porque como no salgo } \\
\text { de mi casa// } \downarrow \text { me la } \\
\text { paso todo el día en la } \\
\text { casa/ todo el día/ o sea } \\
\text { yo me levanto para } \\
\text { comer/ para bañarme y } \\
\text { ya/ no salgo ni nada/ } \uparrow \text { Y } \\
\text { si salgo es porque me } \\
\text { van a buscar a la casa, } \\
\text { no al revés }\end{array}$ \\
\hline
\end{tabular}

Las anteriores respuestas demuestran que los adolescentes participantes se habituaron a vivir en espacios pequeños y que en la habitación de $3 \times 3 \mathrm{~m}^{2}$, el espacio propio, donde pasan el segundo mayor tiempo de sus vidas, siendo el colegio el primero, les proporciona los recursos o distracciones que necesitan. Otras respuestas muestran que los pasatiempos consisten en tener comunicación por redes sociales, ver películas, jugar videojuegos. Otras dos actividades que mostraron una frecuencia importante fueron practicar un deporte y visitar amigos, en el espacio próximo.

Al preguntarles cómo se siente viviendo en una Unidad Residencial, el espacio próximo, respondieron:

A1: $\downarrow$ bueno/ como en una casa

Armando: ¿Por qué?

A1: ...está todo aquí mismo/ tú haces relaciones aquí dentro/ tu consigues amigos aquí dentro/ te distraes acá adentro/ en cambio/ en una casa/ al parecer/ tus amigos están más distanciados/...

A3: ...se me complica un poco/ porque yo no (())/ siempre me ha gustado tener más espacio/ por ejemplo en mi casa nosotros tenemos un patio grandísimo/ terraza/ uno salía/ o sea hacia muchas cosas// en cambio aquí/ es todo más complicado/ pero en parte es bueno/ uno se relaciona con las personas de aquí y es como más seguro ${ }^{\circ}()^{\circ}$ y yy / uno se relaciona// por ejemplo/ yo por mi casa/ $\downarrow^{\circ}()^{\circ}$ mi mamá siempre me decía que era peligroso/ que a ella no le gustaba// en cambio acá uno tiene como que más confianza de que esta gente que está aquí y no van a salir de aquí

A1: aquí en el apartamento/ bueno/ como obviamente es más pequeño/ entonces hay q hacer menos actividades /... Sin embargo, A1 aclara:

Esteban: en el apartamento el espacio no me afecta.

Del diálogo anterior más otra información registrada en las entrevistas se deriva algunas conclusiones sobre lo que significa, para el grupo de adolescentes, vivir en una Unidad Residencial:

1. Es más fácil conseguir amigos

2. Brinda seguridad

3. Hay mayor confianza en las personas

4. Brinda confort

5. Hay libertad para salir al espacio próximo

6. Prefieren perder espacio físico y tranquilidad auditiva, pues consideran que al vivir en un edificio "todo se escucha", mientras se garantice lo descrito en los puntos 1 al 5. 
Compartir habitación, compartir el espacio propio: A diferencia de los niños de 8 a 11 años, los adolescentes hablan de su "propio espacio", además de aspectos relacionados con el orden y la limpieza, que no acostumbran a tener los niños. En algunos casos, se tolera compartir el espacio propio, pero con orden. Esteban, por ejemplo, manifestó lo siguiente:

A1:si/ yo organizo mi cuarto/ el escaparate limpio/ organizado/ vienen y meten la mano en mi escaparate y eso es como si se formara un huracán ahí adentro $\downarrow$...// ...mi hermano está creciendo y no vamos a compartir el cuarto por siempre/ cada uno necesita su cuarto...// ... en el momento/ o sea/ de cambiar de personalidad/va a cambiar sus ideas/y él también va a querer su propio espacio/ su/ su propio closet/ su propia cama//...

Felicidad y ampliación de la espacialidad: El juego aún persiste para los chicos, como el fútbol o micro fútbol, pero en el caso de las chichas, no se registró información sobre la práctica de juego alguno, dentro de la Unidad Residencial. A diferencia de la diversidad de juegos en el rango de 8 a 11 años, indistintamente del género, para la ampliación de la espacialidad en el rango adolescentes es complementado o reemplazado por las conversaciones en el parque, con algunos amigos o amigas de la misma Unidad Residencial, escuchar música, actividad que no fue registrada en el rango de 8 a 11 años, salir a comer a centros comerciales con amigos de la Unidad Residencial o compañeros de clases, disfrutar del cine o jugar en el computador o con video juegos, en el caso de los chicos. Las motivaciones que llevan a los chicos a practicar con los videojuegos son variadas; según Castellana et al. (2006) permiten vivir una aventura en primera persona, donde el adolescente pone en práctica estrategias en un entorno virtual, un espacio virtual, sin consecuencias en la vida real. Malo et al. (2005) refieren que los chicos disponen de videoconsolas con mayor porcentaje en comparación con las chicas. Los chicos parecen muy interesados por este tipo de juego, a través del cual, pueden demostrar las habilidades, la imaginación y la competitividad, mientras que las chichas, indica Figuer et al. (2005), están más interesadas en otro tipo de tecnologías, como el celular, los chats y el correo electrónico. Paralelamente, hay dos razones, por las cuales, los padres comprarían un celular a sus hijos: la primera es la necesidad de control y, la segunda, asegurar una unión con ellos (Kamibeppu \& Sugiura, 2005). Nicolaci-da-Costa (2007) refiere que, desde la perspectiva de las relaciones familiares, el aparato puede servir como herramienta de auto-protección para los jóvenes y de monitorización por parte de los padres para con sus hijos.

Temores a priori a la ampliación de la espacialidad: Se indagó sobre los temores que tienen los adolescentes cuando salen al espacio externo. El rapto no apareció en las res- puestas, como sucedió en el rango de 8 a 11 años (Aroca \& Álvarez, 2016); la prevención hacia el daño de la integridad física fueron las respuestas de mayor frecuencia. También, se evidenció que no tomar la misma ruta de bus para llegar al apartamento generaba temores por el desconocimiento de la trayectoria; las razones por las que determinados espacios son rechazados, son variadas. Se expresa claramente un rechazo a la violencia, real o percibida, que se puede dar en determinadas calles o espacios y que les llevaría a no transitar por ellos o a frecuentar dichos lugares. En el siguiente episodio, se encuentran algunos detalles sobre esta afirmación: A1: Depende para dónde vaya// o sea hay algunos que viven/tengo amigos que viven hasta en Municipio-1/ pero yo para allá no cojo// Entonces cuando...// Me queda muy lejos y ellos quieren ir fiesta de nueve de la noche y yo para venirme a las doce de la noche de allá de Municipio-1 y no me atrevo/ Entonces// normalmente vamos al Buenavista y cojo un bus ahí en la esquina. // ...me han dicho que Municipio-1 es una porquería// Cualquier momento te pueden apuñalar por ahí o algo así// entonces yo mejor no me arriesgo.

Teniendo en cuenta lo anterior y según Ortiz et al. (2014), los adolescentes prefieren los espacios que son reconocidos y aceptados por sus padres y familiares, ya que genera procesos de apropiación del entorno cotidiano.

Temporalidad según los días: Los adolescentes de la investigación pueden salir sin permiso al espacio próximo, a voluntad, pero deben cumplir horarios, los cuales, varían entre el lunes y el jueves y entre el viernes y el domingo; por ejemplo, de domingo a jueves pueden salir en promedio hasta las 8:30 p.m. Los horarios del viernes y sábado son concertados con los padres y, por lo general, es para salir al espacio exterior.

La gran mayoría de adolescentes que hicieron parte de la investigación están en calendario de estudio $B$ y tienen jornadas escolares de 7:00 a.m. a 4:00 p.m. La respuesta de A3 evidencia la saturación del tiempo que esta población tiene de lunes a sábado, más aún la población adolescente:

A3: yo tengo un horario muy variado en toda la semana// Lunes y Martes tengo todo el día en el colegio/ llego tipo 6 y 15// siete de la noche llego a mi casa/ eh// los miércoles tengo en la tarde solamente/ y llego también a las siete y los jueves y los viernes tengo solo en la mañana/ de 6:30 a 12/// $Y$ en eso como me la paso todo el día en el colegio los jueves y o viernes tengo más tiempo como de descanso / porque tengo toda la tarde libre/ y puedo hacer las tareas que me faltaron porque son largas y hacer las tareas del día siguiente y como el sábado tengo clase también / adelantar un poco.

Al preguntarles cuáles son los días más largos que sienten, respondieron de la siguiente forma:

A3: los jueves y los viernes 
Armando: $\uparrow$ ¿̇por qué los jueves y los viernes?//

A3: $\rightarrow$ Largos/ porque siento la tarde. Porque puedo dormir toda la tarde. Yo creo que si estoy durmiendo siempre las horas se pasan más lento y corto, no sé, los sábados

A2: El más largo es el viernes. // 个porque llego del colegio y ya no hago más na'// ya no me estoy distrayendo/ En cambio entre lunes y jueves voy a jugar fútbol/ entonces se va la hora rápido ya// Pero el viernes no/ entonces no sé qué hacer// Me coloco a ver televisión o algunas veces a hablar/ o chateo /o lo que sea//Y se va largo el día//

El viernes tuvo mayor frecuencia como el día más largo, mientras que los fines de semana se consideran que son los días más cortos. Algunas razones de ello son:

A3: ... los sábados las horas se pasan rápido/ y más si uno está haciendo algo// cualquier cosa que puedes estar haciendo/ y cuando vienes a ver ya nueves o diez de la noche ya uno se tiene que ir// $\uparrow$ Pero igual me gustan los sábados porque es donde uno puede hacer/uno puedo salir/ hacer un plan/ hacer algo.

Los adolescentes que viven en apartamentos de pisos superiores prefieren no bajar al espacio próximo de lunes a viernes. Este comportamiento, también se encontró en los niños de rango de 8 a 11 años, es decir, vivir en el primer piso da mayores condiciones de acceso al espacio próximo, pues se siente para ellos como accesible, como un espacio contiguo al propio y al local y que se percibe desde las ventanas de la sala y las habitaciones.

La temporalidad, también se manifiesta por medio del reglamento interno establecido por la Administración de la Unidad Residencial; por ejemplo, el tiempo máximo de uso de la piscina, que es hasta las 6:00 p.m. y los lunes no se puede usar, por mantenimiento. Las chicas reclamaron por el uso de este espacio más no de la piscina, tal como lo manifestó A3:

A3: ... veces hay unos porteros de que nos ven ahí sentados/ hacer nada/ si no sentada sin hacer algo/y no nos dicen nada/ pero/ hay unos como que dicen/ no/ no estén aquí/ y nos regañan a nosotros /...

Confort en los espacios próximo y local: A medida que crecen los niños, por diversas razones que ya se han presentado, el comportamiento grupal o individual de estar en el espacio próximo se tiende a reducir, por circular solo en los espacios locales y propios. Las respuestas de A1 y A3 brindan elementos para dicha afirmación:

A1: $\uparrow$ No/ los fines de semana/// Bueno/ yo no suelo salir// Si es por mí no salgo de mi casa// entonces

Armando: ¿Y por qué?// ¿Por qué no te gusta salir de tu casa?/ ¿El computador?

A1: iSi!
Armando: ¿Y el sábado también te dedicas a qué?// iEn el computador?

A1: iJugar!

Armando: ¿En el computador?

A1: isí!

A3: $\uparrow$ Dormir todo un día// Yo soy de las que puede pasar toda una tarde durmiendo / y si no salgo ni nada// Una vez me pasó así// duré todo el día en mi cuarto/ no salí para nada solo me bañé y me puse la pijama y ya/ Me quedé viendo películas todo el día.

Temores al estar solo en el espacio propio: Veamos el siguiente diálogo:

Armando: a ver/ chan pasado/ por ejemplo/ una noche solos?/ cen el apartamento? cles ha dado miedo?:

A3: no siento nada/ porque/ normalmente me quedo en el cuarto de mi mamá/ en el cuarto de ella donde está el televisor (()) me quedo ahí/ me encierro ahí / hasta esperar a que ellos lleguen/ o si no cuando estoy con mi hermanita/ la menor/ nos quedamos las dos y yo le digo/ bueno/ ya sabes lo que tienes que hacer y ella se queda dormida ahí/ me toca cuidarla a ella// pero temor así/ de que nos vaya a pasar algo/ no.

A2: iyo sí! //...a veces si// yo veo películas de miedo/ §: (Risas) / entonces/ me quedo con la idea y ajá/ entonces me paro de la sala ahí/ miro pa' allá/ pa' el cuarto/ pa' el fondo/ si me va a salir algo/entonces no.

Al igual que en el rango de 8 a 11 años, los adolescentes también manifestaron miedos de quedarse solos en los espacios locales, los apartamentos. Para Cabello et al. (2006), el miedo constituye un sistema de alarma primitivo, que permite al niño evitar situaciones que pueden llegar a ser potencialmente peligrosas. Se trata de respuestas normales, adaptativas, especialmente, comunes en la infancia y la adolescencia.

Compartir el espacio próximo: En Aroca \& Álvarez (2016), se evidenció que la percepción de los niños de 8 a 11 años es que los adolescentes los excluyen en las zonas de juego que son comunes, como la cancha de micro fútbol que, a la vez, es de baloncesto, pero al escuchar las respuestas de los adolescentes, se presentó una versión contraria, más de inclusión o respeto. Las siguientes respuestas de A2 y A3 respaldan lo anterior:

A2: a veces pa' no/ a veces pa' no sácalos/ como somos grandes ya/ nos ponemos a jugar/ el coge a un chiquito/ yo cojo a un chiquito / y así/ a jugar/ a jugar pareja/ porque si no/ juego con ellos/ no podemos jugar porque/ siempre vaya a salir llorando/.

A3: no/ si ii/ por ejemplo/ a veces que jugamos voleibol/ nos vamos para la cancha/nosotras si/ vemos a alguna niña jugando/ nosotras como que nos corremos un poquito/ o le damos espacio. 
Recorrido por el sótano o parqueadero subterráneo: Esperábamos que el sótano o parqueadero subterráneo sería un lugar del espacio próximo que no frecuentaran los adolescentes, pero notamos que lo usan más que los niños de 8 a 11 años, para montar bicicleta, como corredor o espacio de juego cuando está lloviendo o para montar la patineta; sin embargo, algunas de estas actividades no son consideradas como juego, por parte de ellos. Al parecer el juego significa que otras personas se vinculen a la actividad, actividades solitarias de montar la patineta o la bicicleta, no es para ellos jugar.

Cambio de vivienda: de un apartamento a una casa: Al preguntarles a los adolescentes qué perderían o ganarían si se mudan a una casa, en un barrio residencial, se pudo clasificar lo siguiente. Pierden: los amigos, seguridad, tiempo para entrenos deportivos, los vecinos, la confianza de los padres al salir a otra casa que no sería lo mismo de un apartamento a otro, menos confort en el sentido de que en una casa son más los oficios por hacer. Ganan: espacio y comodidad en el cuarto.

Como se puede notar, según lo adolescentes entrevistados, se pierden más aspectos de la vida cotidiana que lo que se gana, al mudarse de un apartamento a una casa.

Ampliación de la espacialidad y temporalidad en función de las relaciones con los padres: Se evidenciaron dos tipos de comportamientos de los padres. El primero, que condiciona que la persona con quien va a salir el adolescente fuera de la unidad residencial debe ser alguien conocida por ellos $y$, el segundo, no podemos decir que se trata de un comportamiento de indiferencia sino, tal vez, de confianza a los hijos, para que ellos amplíen su espacialidad.

Como lo refiere Parra Jiménez \& Oliva (2006) y Collins \& Steinberg (2006) existe una disminución en los niveles de control, que padres y madres ejercen sobre sus hijos, a medida que transcurre la adolescencia, siendo esta disminución uno de los principales reajustes que los padres suelen realizar en su estilo parental, para adaptarse a la mayor madurez de su hijo adolescente y a sus nuevas necesidades; no obstante, se presentan conflictos entre padres y madres e hijos o hijas por el incumplimiento de los horarios. El cumplimiento de horarios es la base de la confianza entre ellos. Los adolescentes manifestaron su deseo de estar más tiempo en el espacio externo a la Unidad Residencial, que retornar al encierro y monotonía del espacio local y propio. Algunas respuestas fueron:

A3: Ee si puedo/ con tal de que mi mamá los conozca/ si puedo// Pero mientras mi mamá no sepa de la existencia de mis amigos de afuera no me deja.

A2: Joda yo ee// (Entre risas) ido a las Mercedes/ a Villa Campestre/// a/ al Recreo.
A4: Si/ mi mamá confía que/ si yo le digo voy para una $\downarrow$ parte es para esa parte, ella me ha dado mucha confianza para ir.

Para finalizar, en el rango de 14 a 16 años, se evidenció que los padres daban los primeros permisos a sus hijas o hijos para quedarse a dormir en casa o apartamento de amigos o amigas, ubicadas en el espacio externo. Lo particular de ello era que las chichas han tenido más permiso de quedarse en "casa ajena" que los chicos; sin embargo, en los tiempos libres, los padres consideran que es mejor que las chichas permanezcan en el apartamento y los chicos salieran del apartamento:

A3: Porque mi mamá no le gusta que yo pase mucho tiempo fuera de la casa// Siempre me dice que mira que yo tan callejera/ ósea/ nunca le ha gustado eso// O que yo duerma fuera de mi casa// Tampoco le ha gustado/ tiene que ser donde mi papá o que yo le diga días antes que voy para donde una amiga o algo// Pero no le gusta eso.

Los niños o adolescentes, progresivamente, tienden a permanecer enclaustrados en su espacio propio o local. Son regularmente los padres quienes los motivan a salir. El siguiente episodio así lo respalda.

A1: Depende// Porque si está la casa sola yo juego en el computador todo el día/ si está la casa sola/ pero/ ajá como la gente se aburre de verme ahí sentado todo el día me mandan a levantar// entonces unas tres/ cuatro horas// Y iya!

Armando: ¿'Tú mamá te regaña por eso o tu papá?

A1: Los dos

Armando: ¿qué te dicen?

A1:no pierda su tiempo// aproveche y salga más y cosas así Armando: ¿y te dicen salga?

A1:si/ adolescentes normales los obligan a estar en la casa// a mí me obligan a salir.

Collins \& Laursen (2004) consideran que en periodos de rápidos cambios evolutivos, como la transición a la adolescencia, las expectativas de los padres con respecto al comportamiento de sus hijos son violadas con frecuencia, lo que causará conflictos y malestar emocional. De otra forma, los adolescentes que viven en Copropiedad Horizontal manifiestan y actúan por ampliar su temporalidad y espacialidad, al querer conocer cada vez más el espacio externo, lo que conlleva a generar conflictos con sus padres.

En la relación con los padres y la ampliación de la temporalidad, las reglas que se establecen responden a pautas de tiempo. En medio de esas reglas de tiempo está el uso adecuado de las llaves del apartamento; es un acto de confianza de los padres a los hijos, para que ellos amplíen su temporalidad y su espacialidad. En el rango de 8 a 11 años, la llave del apartamento aparece como un objeto que representa el 
derecho a ampliar la espacialidad y la temporalidad (Aroca \& Álvarez, 2016).

Para finalizar, el espacio próximo, lo interno en la Unidad Residencial y externo al apartamento es una zona, en particular, el parque, que se emplea como lugar de encuentro de amigos, para planeación de actividades, mientras que, para el rango de 8 a 11 años, el espacio próximo es una zona de diversos juegos; en los adolescentes, lo que se evidenció fue mayor enclaustramiento en los espacios local y propio. Los adolescentes participantes de la investigación manifestaron su interés por ampliar su espacialidad y temporalidad en el espacio externo a la Unidad Residencial, lo que, en algunas circunstancias, le genera conflicto con los padres de familia, sea por el cumplimiento de horarios o lugares a visitar o conocer. El espacio propio, la habitación, prefieren no compartirlo con hermanos y tanto el espacio propio como el local, el apartamento, son lugares que satisfacen sus necesidades de comunicación virtual, la virtualización de la amistad, juegos virtuales, por lo general, teniendo como oponente al software o participantes desconocidos

Conflictos de intereses: El manuscrito fue preparado y revisado con la participación de todos los autores, quienes declaramos que no existe conflicto de intereses que ponga en riesgo la validez de los resultados presentados.

\section{BIBLIOGRAFÍA}

1. ALFONSO, Ó. 2005. La residencia en condominios en un ámbito metropolitano andino. La conquista del campo por los citadinos y el orden segmentado en la región Bogotá, Cundinamarca. En: Goueset, V.; Cuervo, L.; Lulle, T.; Coing, H., (eds.). Hacer metrópoli. La región urbana de Bogotá de cara al siglo XXI. Universidad Externado de Colombia (Bogotá). p.235-290.

2. AROCA, A.; ÁlVAREZ, L. 2016. Condiciones témporo-espaciales de niños que habitan en una copropiedad horizontal de la Ciudad de Barranquilla, Colombia. Rev. U.D.C.A Act. \& Div. Cient. (Colombia). 19(2):479-487.

3. BLANCO, J. 2007. Espacio y territorio: elementos teórico-conceptuales implicados en el análisis geográfico. En: Fernández, M.V. \& Gurevich, R. (Eds.) Geografía. Nuevos temas, nuevas preguntas. Un temario para su enseñanza. Editorial Biblios (Argentina). p.37-64.

4. CABELLO, V.E.; GONZÁLEZ, S.; ALONSO, V.; GUILLÉN, J.L.; GARRIDO, L.E.; IRURTIA, M.J. 2006. Los mie- dos infantiles: Un análisis por edades y sexo. Revista Humanitas. (Alicante). 3:16-22.

5. CASTELLANA, M.; SÁNCHEZ-CARBONELL, X.; BERANUY, M.; GRANER, C. 2006. La relació de l' adolescent amb les TIC: Un tema de rellevància social. Full informatiu del col-legi oficial de psicòlegs de Catalunya. (Catalunya). 192:22-23.

6. COLLINS, W.A.; LAURSEN, B. 2004. Parent-adolescent relationships and influences. En: Lerner, R.M. \& Steinberg, L. (eds.). Handbook of adolescent psychology. Willey (New Jersey). p.331-361.

7. COLLINS, W.A.; STEINBERG, L. 2006. Adolescent development in interpersonal context. En: Eisenberg, N. (ed.). Social, emotional, and personality development. Handbook of Child Psychology. Wiley (New York). p.1003-1067.

8. FIGUER, C.; GONZÁLEZ, M.; MALO, S.; CASAS, F. 2005. El món adolescent en l'entorn de l'ús de l'ordinador i Internet. Perspectiva Escolar. 299:36-41.

9. GARCÍA, B.Y.; GUERRERO, J. 2012. Núcleos de Educación Social - NES. Investigación, Prevención y Participación. Universidad Distrital Francisco José de Caldas (Colombia). 144p.

10. GARCÍA, F.A.; PERALTA, M.D. 2015. Urbanizaciones cerradas y su vinculación con el espacio exterior en la ciudad de Cali: una propuesta metodológica para su análisis. Prospectiva (Colombia). 19:197-221.

11. GARRIDO, M. 2005. El espacio por aprender, el mismo que enseñar: las urgencias de la educación geográfica. Cad. Cedes (Brasil). 25(66):137-163.

12. JANOSCHKA, M.; BORSDORF, A. 2006. Condominios fechados and barrios privados. The rise of private residential neighborhoods in Latin America. En: Georg, G.; Webster, C.; Klaus, F. (eds.). Private cities. Global and local perspectives. Routledge (New York). p.92-108.

13. KAMIBEPPU, K.; SUGIURA, H. 2005. Impact of the mobile phone on junior hight-school students' friends hips in the Tokyo metropolitan area. Cyberpsychology \& Behavior. 8(2):121-130.

14. KIM, Y.; KIM, E.; KANG, J. 2003. Teen's mall shopping motivation: functions of loneliness and media usage. Family and Consumer Sciences Research J. 32(2):140-167. 
15. LASARTE, C.; FELIU, M.I. 2005. Comunidades de vecinos y administración de fincas urbanas y complejos residenciales. TECNOS (España). 1026p.

16. MALO, S., FIGUER, C.; GONZÁLEZ, M.; CASAS, F. 2005. El telèfon mòbil: un ràpid canvi tecnològic $\mathrm{i}$ també de relació. Perspectiva Escolar. 299(1):28-35.

17. MELLA, O. 2000. Grupos Focales ("Focus Groups"). Técnica de investigación cualitativa. Documento de trabajo No. 3, Cide, Santiago, Chile. Disponible desde Internet en: http://biblioteca.uahurtado.cl/ ujah/856/txtcompleto/txt105091.pdf (con acceso 04/02/2016).

18. MEYER, K.; BÄRH, J. 2004. La difusión de condominios en las metrópolis latinoamericanas. El ejemplo de Santiago de Chile. Rev. Geografía Norte Grande (Chile). 32:39-53.

19. NICOLACI-DA-COSTA, A. M. 2007. Celulares: um "presente do céu" para as mães de jovens. Psicologia e Soc. (Belo Horizonte). 19:108-116.

20. ORTIZ, A.; PRATS, M.; BAYLINA, M. 2014. Procesos de apropiación adolescente del espacio público: otra cara de la renovación urbanística en Barcelona. Boletín de la Asociación de Geógrafos Españoles (España). 65:37-57.
21. PARRA JIMÉNEZ, A.; OLIVA, A. 2006. Un análisis longitudinal sobre las dimensiones relevantes del estilo parental durante la adolescencia. Infancia y Aprendizaje (España). 29(4):453-470.

22. PIEDRAHÍTA, V.; CEBALLOS, L.M.; BEJARANO, $M$. 2012. La Moda y el comportamiento adolescente urbano en centros comerciales de Medellín. AD-minister (Colombia). 20:45-61.

23. RINCÓN, M.T.; MALDONADO, M.C. 2007. Acerca de los motivos para vivir en espacios residenciales multifamiliares. Prospectiva. 12(1):147-180.

24. ROITMAN, S. 2003. Barrios cerrados y segregación social urbana. Scripta Nova. Revista electrónica de geografía y ciencias sociales (España). 7(146). Disponible desde Internet en: http://www.ub.es/geocrit/ sn/sn-146(118).html (con acceso 01/10/2017).

25. VASCO, C. 2000. El tiempo en la teoría general de procesos y sistemas. En: Lopera, J. (ed.). El problema del tiempo. Universidad nacional de Colombia, Sede Medellín (Medellín). p. 215-240.

Recibido: Octubre 18 de 2017

Aceptado: Mayo 7 de 2018

\section{Cómo citar:}

Aroca Araújo, A.; Álvarez Toro, L.; Mejía Pérez, M.A. 2018. Condiciones témporo-espaciales de adolescentes que habitan en una copropiedad horizontal de la ciudad de Barranquilla, Colombia. Rev. U.D.C.A Act. \& Div. Cient. 21(1): 217-225. 